\title{
BIENESTAR PSICOLÓGICO, ASERTIVIDAD Y RENDIMIENTO ACADÉMICO EN ESTUDIANTES UNIVERSITARIOS SANMARQUINOS
}

\author{
Carlos Velásquez C. ${ }^{1}$, Willam Montgomery U., Victor Montero L., Ricardo Pomalaya V., \\ Alejandro Dioses Ch., Natalia Velásquez C.a, Raúl Araki O., Deivit Reynoso E. \\ Universidad Nacional Mayor de San Marcos, Lima, Perú \\ (RECiBido El 14/10/2008, ACEPTADO EL 19/12/2008)
}

\begin{abstract}
RESUMEN
En esta investigación se examinan las relaciones entre el bienestar psicológico, la asertividad y el rendimiento académico en los estudiantes de Facultades representativas de las diversas áreas de estudio de la Universidad de San Marcos. Para ello, se utilizó la Escala de Bienestar Psicológico de Ryff (que mide relaciones positivas, autonomía, dominio del entorno, crecimiento personal y propósito en la vida), el Inventario de Asertividad de Rathus y el rendimiento académico. El estudio es de tipo descriptivo-correlacional, con un muestreo no probabilístico circunstancial de la población a estudiada. Los datos fueron procesados con el paquete estadístico SPSS (Statistical Package for the Social Sciencies), realizando un análisis de correlación y llegando a encontrarse que sí existe correlación significativa, $\mathrm{p}<0.05$, entre las variables de estudio, aceptando la hipótesis general planteada. Además, se encontró que existe relación significativa entre el bienestar psicológico y la asertividad, tanto en varones como en las mujeres. De igual manera, en todas las facultades la relación es significativa entre las variables en estudio, excepto en la Facultad de Ingeniería Industrial donde la relación entre asertividad y rendimiento académico no resultó ser significativa.
\end{abstract}

Palabras clave: Bienestar psicológico, asertividad, rendimiento académico, contexto universitario.

\begin{abstract}
In this investigation the relations between the psychological well-being are examined, the assertiveness and the academic yield in the students of representative Faculties of the diverse areas of study of the University of San Marcos. For it, the Scale of Psychological Wellbeing of Ryff (that measures positive relations, autonomy, dominion of the surroundings, personal growth and intention in the life), the Inventory of Asertividad de Rathus and the academic yield were used. The study is of corelational descriptive type, with a circumstantial nonprobabilística sampling of the studied population to. The data were process with statistical package SPSS (Statistical for Package the Social Sciencies), making a correlation analysis and getting to be that yes significant correlation exists, $\mathrm{p}<0.05$, between the study variables, accepting the raised general hypothesis. In addition one was that significant relation between the psychological well-being and the assertiveness exists, as much in men as in the women. Of equal way in all the faculties the relation is significant between the variables in study,
\end{abstract}

1 Docente Principal de la Facultad de Psicología de la UNMSM. E-mail: cvelasquezcenteno@hotmail.com 
except in the faculty of industrial engineering the relation between assertiveness and academic yield did not turn out to be significant

Keywords: Psychological Well-being, assertiveness, academic performance, university context.

\section{INTRODUCCIÓN}

En un mundo fuertemente competitivo, los estudiantes universitarios deben enfrentar el embate de una serie de demandas sociales y académicas que el medio circundante les exige, y que a veces exceden la capacidad de los jóvenes para estar a la altura de las circunstancias. En esta etapa de la vida, los alumnos universitarios no sólo están sometidos a la evaluación continua de sus maestros y autoridades, sino también de los miembros del grupo estudiantil al cual pertenecen y con el que se relacionan cotidianamente. Todo ello los obliga a establecer y consolidar su posición dentro de ese espectro institucional en su existencia concreta, y a prepararse también para soportar sentimientos negativos en caso de no poder responder del modo social y lectivamente esperado.

En este contexto, los repertorios conductuales de asertividad y los sentimientos de bienestar subjetivo son importantes para los individuos. Las competencias asertivas permiten un adecuado manejo interpersonal de situaciones sociales, y los sentimientos de bienestar aportan la dosis de tranquilidad y optimismo suficientes como para desarrollar estrategias de afronte a los problemas cotidiaños, en especial los referentes a la franja ocupacional del estudiante común y corriente: la dedicación lectiva. La calidad y cantidad del rendimiento académico vienen a ser buenos referentes del éxito que el joven alumno universitario consigue, partiendo de sus capacidades para evaluar en general positivamente las situaciones (autorreportar "sentirse bien") y manejar de manera adecuada las relaciones interpersonales.

Por ello es que en el sistema curricular de la carrera profesional de Psicología en la Universidad Nacional Mayor de San Marcos (2006) se mencionan puntualmente las competencias sociopersonales, aunadas a las lectivas, como parte integrante del perfil del ingresante y del egresado. En general, esto es reconocido por todos los expertos (Van-Der Hofstadt, Gómez, Alarcón y García, 2006).

Es, pues, relevante ocuparse de las relaciones entre los aspectos mencionados, teniendo en cuenta que el avance del conocimiento de los diversos factores que inciden el rendimiento académico, sirve para precisar la adopción de políticas educativas y la toma de decisiones referidas a la articulación de estrategias, programas y capacitaciones, abordando de manera integral la situación de los educandos.

Siguiendo esos lineamientos, la presente investigación examina empírica y conceptualmente las interacciones entre las variables de bienestar psicológico, asertividad y rendimiento académico en los estudiantes de facultades representativas de las diversas áreas de estudio de la Universidad Nacional Mayor de San Marcos: Medicina (Ciencias de la Salud), Derecho y Educación (Humanidades y Ciencias Sociales), Matemáticas (Ciencias Básicas), Ingeniería Industrial (Ingenierías), y Administración (Económica Empresarial). Para ello, se relacionan la Escala de Bienestar Psicológico de Ryff (que evalúa factores como 
relaciones positivas, autonomia, dominio de entorno, crecimiento personal y proposito en la vida), el Inventario de Asertividad de Rathus, y el rendimiento acádemico expresado en el promedio de calificaciones (tercio superior e inferior) alcanzado en las diversas materias de estudio.

Es así que se formula el problema de la siguiente manera: ¿Cuáles son las relaciones entre el bienestar psicológico, la asertividad, y el rendimiento académico, en función a la edad, sexo y facultad de procedencia? En función a lo dicho, se busca determinar si existe relación entre el nivel de bienestar psicológico y el grado de asertividad, entre el nivel de bienestar psicológico y el nivel de rendimiento académico, y entre el grado de asertividad y el nivel de rendimiento académico. El texto constituye una adaptación del informe de investigación originalmente presentado al Instituto de Investigaciones Psicológicas, por lo que para mayores detalles se remite a lo consignado allí.

\section{Definición de conceptos}

\section{Bienestar psicológico}

Aunque la investigación del constructo de bienestar ha sido un tanto confusa -mezclándose nociones como bienestar psicológico, felicidad, disfrute y particularmente calidad de vida-, el bienestar psicológico puede definirse de manera general como un concepto ligado a la percepción subjetiva que tiene un individuo respecto a los logros conseguidos por él, y su grado de satisfacción personal con sus acciones pasadas, presentes y futuras. En tal sentido, refleja el sentir positivo y el pensar constructivo de la persona para consigo mismo (Diener, Suh, Lucas y Smith, 1999).

En opinión de García y González (2000), el bienestar psicológico se diferencia claramente de la calidad de vida. De hecho, ésta incluye el concepto de bienestar, puesto que implica una interacción bastante compleja entre factores objetivos ligados a condiciones externas de tipo económico, sociopolítico, cultural y ambiental, mientras que el bienestar está signado por la autovaloración vital que hace la persona en función de su nivel individual de satisfacción.

Tal como señalaron Ryan y Deci en 2001 (cit. por Díaz, Rodríguez, Blanco, Moreno, Gallardo, Valle y Van Dierendonck, 2006, p. 572), los estudios tradicionales acerca del bienestar se han perfilado en dos líneas: una vinculada con la felicidad como indicadora de la calidad de vida, y otra ligada al desarrollo del potencial humaño.

De un tiempo a esta parte, se han hecho varias investigaciones en Iberoamérica sobre el bienestar, abarcando aspectos tales como la personalidad (Díaz y Sánchez, 2002; Fierro, 2006), el disfrute (Padrós, 2002), el orden social y la salud mental (Blanco y Díaz, 2005), y el afrontamiento (Salotti, 2006). En todos esos estudios se han identificado correlaciones positivas entre las variables seleccionadas. Tras el concepto postulado por Diener en los años noventa, también se entiende el bienestar en su sentido cognitivo como satisfacción con la vida (Atienza, Pons, Balaguer, y García-Merita, 2000). Así, Tarazona (2005) aplica en nuestro medio la Escala de Satisfacción con la Vida de Diener y colaboradores en escuelas pública y privada, encontrando correlación entre la autoestima y el grado de 
satisfacción, ocurriendo que en ambas variables los varones alcanzan un nivel más alto que las mujeres en la escuela privada, mientras que el resultado entre los hombres y mujeres de escuela pública muestra un bajo nivel.

También Inga y Vara (2006) hacen un estudio sobre los factores asociados a la satisfacción de vida de adultos mayores de 60 años en Lima: resentimiento, satisfacción de vida, maltrato, dependencia, apoyo social, identidad amical prosocial e identidad amical antisocial. Sus resultados muestran alta satisfacción vital; mayor nivel de insatisfacción en debilidad corporal, sentimientos de ser abandonados por sus familiares, así como soledad o desesperanza; el resentimiento fue el factor más asociado a la satisfacción de vida, seguido del nivel de instrucción, el apoyo social recibido, el consumo de sustancias psicoactivas, el maltrato familiar y la densidad amical.

\section{Asertividad}

Wolpe (Cf.: Delgado Senior, 1983) dice que una aserción "es toda expresión socialmente aceptable de derechos y sentimientos personales, lo cual incluye rechazos, reclamos, expresiones de premio y afecto, y exclamaciones de sentimientos personales tales como satisfacción, disfrute y rabia" (p. 133). Lange (1980/1983), por su parte, acota que es "la comunicación de las opiniones, creencias, sentimientos y deseos personales de una forma directa, honrada y adecuada" (p. 310). Es, pues, una actitud intermedia entre las actitudes pasiva y agresiva frente a otras personas, que comprende factores de conducta verbal (fluidez, tono, vocabulario) y no verbal (postura corporal, gestos, expresión facial).

Las habilidades sociales implicadas en el comportamiento asertivo se adquieren por aprendizaje (observación, imitación, información, ensayo), acrecientan el reforzamiento social que recibe el individuo y son afectadas por la especificidad de las situaciones, la edad, el sexo y el status. La asertividad es hoy un concepto popular y una aspiración de toda terapia de afrontamiento, así como, una vez desarrollada, una especie de "repertorio preventivo" contra la ansiedad. Sus componentes no son inespecíficos y por eso constituye un objeto abordable para el trabajo conductual, pero no debe esperarse que el entrenamiento en las habilidades que le son propias convierta al individuo en un "cronómetro" preparado para responder "adecuadamente". Es necesario percatarse de que la posesión de repertorios sociales asertivos no obliga -ni necesariamente capacita- a la persona para ser siempre asertiva. Juega un papel importante el balance "costo-beneficio" que, en función a las conveniencias eventuales, suele estar presente en la evaluación (basada en una historia personal y en las disposiciones individuales) que cada sujeto hace de cómo puede o debe responder según las conveniencias del momento (Montgomery, 1999).

El uso de este constructo disposicional se ha extendido a gran cantidad de ámbitos, desde la simple vulgarización (Alberti y Emmons, 1974/2006; Fensterheim y Baer, 1975/2001) hasta la especialización en tareas educativas (Pérez, 2000), pasando por la enseñanza de valores (García, 1999), la terapia marital, la esquizofrenia crónica y la desviación sexual, entre otros. 


\section{Rendimiento académico}

Pizarro (1985) y Novaez (1986) coinciden en definir el rendimiento académico como el conjunto de ratios efectivos obtenidos por el individuo en determinadas actividades académicas, como respuesta a un proceso de instrucción o formación interpretable de acuerdo con objetivos o propósitos educativos antes fijados. En tal sentido, son indicadores de competencias aptitudinales, ligadas además a factores volitivos, afectivos y emotivos. Tales competencias reflejan el grado de logro de los objetivos establecidos en los programas oficiales de estudio.

El rendimiento académico puede ser afectado por muchas variables. Aliaga (2001) estudia algunas de ellas en la Universidad Nacional Mayor de San Marcos, concluyendo que existe relación significativa con la motivación y las estrategias de aprendizaje.

Reyes (2003), de la misma institución, indica que en su muestra sólo la octava parte de los estudiantes alcanzó un rendimiento académico satisfactorio de los contenidos de primer año, y la mitad posee un nivel bajo de asertividad. El rendimiento académico del grupo estudiado correlaciona positivamente con las modalidades de autoconcepto académico y familiar, encontrándose además una correlación negativa con los rasgos de personalidad sumisión-dominancia.

Por otra parte, García (2005) investiga la relación entre las habilidades sociales y el clima social familiar con el rendimiento académico en estudiantes universitarios de Lima Metropolitana de las Universidades San Martín de Porres y San Marcos, en base a la Lista de Chequeo de Habilidades Sociales de Goldstein y la escala de clima social en la familia de Moos y Trickett. Los resultados arrojaron una correlación positiva y significativa entre habilidades sociales y el clima social en la familia, y no existe estadísticamente una correlación significativa entre habilidades sociales y clima social en la familia con el rendimiento académico.

Fragoso y Alcántara (2005) muestran, asimismo, la relación entre factores estresantes y rendimiento académico en alumnos de secundaria mexicaños, mediante la prueba de sucesos de vida, encontrando que las variables que más afectan a los de bajo rendimiento son la familiar y personal y a los de alto rendimiento, los problemas de conducta.

En cuanto a diferencias de género, Grande, Medina, Gomá y Valero (1998) ubican un mejor rendimiento académico de las mujeres sobre los varones en el área humanística, asimismo son más sociables, más impulsivas y disciplinadas, pero menos sensibles que los varones. En otra investigación (Medina, Grande, Gomá y Valero 1998) se ve que los varones se decantan más por el área científico-técnica, y que no aparecen diferencias en las áreas de Derecho y Economía.

\section{MÉTODO}

\section{Diseño}

El estudio es de tipo descriptivo-correlacional, con un muestreo probabilistico aleatorio de la población analizada. Los instrumentos utilizados fueron analizados psicométricamente 
para adecuarlos a nuestra realidad. Para el análisis estadístico principal, se utilizó el método del Producto Momento de Pearson. Para la interpretación estadística, se hace uso de la estadística descriptiva, presentando comparaciones cuantitativas y cualitativas para, finalmente, efectuar la interpretación y explicación general.

\section{Participantes}

La muestra está conformada por 1244 alumnos que comprenden sexos masculino (551) y femenino 693) de facultades representativas de las diversas áreas de estudio de la Universidad Nacional Mayor de San Marcos, comprendiendo Medicina (Ciencias de la Salud), Derecho y Educación (Humanidades y Ciencias Sociales), Matemáticas (Ciencias Básicas), Ingeniería Industrial (Ingenierías), Administración (Económica Empresarial). La distribución muestral figura en la tabla 1.

Tabla N. ${ }^{\circ}$ 1. Sujetos del estudio.

\begin{tabular}{|c|c|c|c|c|c|c|c|c|c|}
\hline & & & \multicolumn{6}{|c|}{ Facultad } & \multirow[b]{2}{*}{ Total } \\
\hline & & & $\begin{array}{c}\text { Medicina } \\
\text { Humana }\end{array}$ & Derecho & Educación & Matematica & $\begin{array}{l}\text { Ingeniería } \\
\text { Industrial }\end{array}$ & Administración & \\
\hline \multirow[t]{4}{*}{ Sexo } & Masculino & N. ${ }^{o}$ de alumnos & 129 & 60 & 37 & 151 & 85 & 89 & 551 \\
\hline & & $\%$ & $23.4 \%$ & $10.9 \%$ & $6.7 \%$ & $27.4 \%$ & $15.4 \%$ & $16.2 \%$ & $100.0 \%$ \\
\hline & Femenino & N. ${ }^{\circ}$ de alumnos & 298 & 69 & 83 & 78 & 35 & 130 & 693 \\
\hline & & $\%$ & $43.0 \%$ & $10.0 \%$ & $12.0 \%$ & $11.3 \%$ & $5.1 \%$ & $18.8 \%$ & $100.0 \%$ \\
\hline \multirow[t]{2}{*}{ Total } & & N. ${ }^{\circ}$ de alumnos & 427 & 129 & 120 & 229 & 120 & 219 & 1244 \\
\hline & & $\%$ & $34.3 \%$ & $10.4 \%$ & $9.6 \%$ & $18.4 \%$ & $9.6 \%$ & $17.6 \%$ & $100.0 \%$ \\
\hline
\end{tabular}

\section{Instrumento}

1. Escala de Bienestar Psicológico de Ryff (Díaz, Rodríguez, Blanco, Moreno, Gallardo, Valle y Van Dierendonck, 2006), que evalúa las escalas de relaciones positivas, autonomía, dominio del entorno, crecimiento personal y propósito en la vida. Su calificación está dada a partir de puntuaciones entre 1 (totalmente en desacuerdo) y 6 (totalmente de acuerdo).

2. Inventario de Asertividad de Rathus (Rathus, 1980), que analiza el problema de la medición de la asertividad, a través de un inventario de 30 ítems para evaluar el constructo. Su calificación está dada a partir de una clave de puntuación desde +3 (muy característica, extremadamente descriptiva), hasta -3 (definitivamente no característica, nada descriptiva).

\section{Procedimiento}

El estudio se llevó a cabo en diversas Facultades de las áreas de estudio que ofrece la Universidad Nacional Mayor de San Marcos, comprendiendo a alumnos del Primero al Décimo ciclo. Se conformó tres grupos, el primero integrado por alumnos cuyo promedio general es el más alto (tercio superior), el segundo grupo fue integrado por alumnos del 
tercio medio, y el tercer grupo formado por los alumnos del tercio inferior. Se aplicó las escalas de bienestar psicológico y de asertividad dentro del salón de clases de cada uno de los grupos de las Facultades mencionadas.

Se correlacionó las variables con el rendimiento académico mediante pruebas de asociación para variables ordinales, coeficientes de correlacion Producto Momento de Pearson y Análisis de Varianza No Paramétrica, con lo cual se estableció las diferencias en función de las características de las puntuaciones obtenidas. Para la caracterización de las variables psicológicas, se utilizó la Estadística Descriptiva. Los cuadros y gráficas se elaboraron empleando el Word 95 para Windows.

\section{RESULTADOS}

Los datos fueron procesados con el paquete estadístico SPSS (Statistical Package for the Social Sciencies), llegando a encontrarse que existe correlación significativa positiva, como se muestra en la tabla $2, \mathrm{p}<0.05$, entre el bienestar psicológico y la asertividad en términos generales, y aunque no se está estableciendo una relación causal, puede afirmarse que un aumento en una de las variables corresponde un aumento en la otra variable.

Tabla N. ${ }^{2}$ 2. Coeficiente de correlación.

\begin{tabular}{llcc}
\hline & & Asertividad & $\begin{array}{c}\text { Bienestar } \\
\text { psicológico }\end{array}$ \\
\hline Asertividad & Correlación de Pearson & 1 & $.374^{* *}$ \\
& Sig. (bilateral) & & .000 \\
& $\mathrm{~N}$ & 1203 & 1193 \\
Bienestar psicológico & Correlación de Pearson & $.374^{* *}$ & 1 \\
& Sig. (bilateral) & .000 & \\
& $\mathrm{~N}$ & 1193 & 1230 \\
\hline
\end{tabular}

** La correlación es significativa al nivel 0,01 (bilateral).

Además se encontró que existe relación significativa positiva $(r=0.404, p<0.05)$ entre el bienestar psicológico y la asertividad, tanto en varones como en mujeres.

Tabla N. ${ }^{\circ}$ 3. Correlaciones.

\begin{tabular}{lllcc}
\hline Sexo & & Asertividad & $\begin{array}{c}\text { Bienestar } \\
\text { psicológico }\end{array}$ \\
\hline Masculino & Asertividad & Correlación de Pearson & 1 & $.404 * *$ \\
& & Sig. (bilateral) & & .000 \\
& & $\mathrm{~N}$ & 542 & 539 \\
& \multirow{3}{*}{ Bienestar psicológico } & Correlación de Pearson & $.404 * *$ & 1 \\
& & Sig. (bilateral) & .000 & \\
& & $\mathrm{~N}$ & 539 & 547 \\
& & Correlación de Pearson & 1 & $.349 * *$ \\
& Asertividad & Sig. (bilateral) & & .000 \\
& & $\mathrm{~N}$ & 661 & 654 \\
& & Correla ción de Pearson & $.349 * *$ & 1 \\
& Bienestar psicológico & Sig. (bilateral) & .000 & \\
& & $\mathrm{~N}$ & 654 & 683 \\
\hline
\end{tabular}

** La correlación es significativa al nivel 0,01 (bilateral). 
Con respecto a la edad, esta relación también es positiva y altamente significativa tanto en los adolescentes como en los jóvenes, y apenas significativa en los adultos $(r=0.245, p=$ 0.041); por lo que se observa que, de acuerdo a los valores del coeficiente de correlación $r$ con el avance en la edad de los sujetos, la asociación entre el bienestar psicológico y la asertividad se va haciendo más débil.

Tabla N. ${ }^{\circ}$. Correlaciones.

\begin{tabular}{|c|c|c|c|c|}
\hline \multicolumn{3}{|c|}{ Edad (Categorizada) } & \multirow{2}{*}{$\begin{array}{c}\text { Asertividad } \\
1\end{array}$} & \multirow{2}{*}{$\begin{array}{r}\begin{array}{r}\text { Bienestar } \\
\text { psicológico }\end{array} \\
.414^{* *}\end{array}$} \\
\hline \multirow[t]{6}{*}{ Adolescente } & Asertividad & Correlación de Pearson & & \\
\hline & & Sig. (bilateral) & & .000 \\
\hline & & $\mathrm{N}$ & 137 & 136 \\
\hline & Bienestar psicológico & Correlación de Pearson & $.414 * *$ & 1 \\
\hline & & Sig. (bilateral) & .000 & \\
\hline & & $\mathrm{N}$ & 136 & 142 \\
\hline \multirow[t]{6}{*}{ Joven } & Asertividad & Correlación d e Pearson & 1 & $.382 * *$ \\
\hline & & Sig. (bilateral) & & .000 \\
\hline & & $\mathrm{N}$ & 996 & 987 \\
\hline & Bienestar psicológico & Correlación de Pearson & $.382 * *$ & 1 \\
\hline & & Sig. (bilateral) & .000 & \\
\hline & & $\mathrm{N}$ & 987 & 1013 \\
\hline \multirow[t]{6}{*}{ Adulto } & Asertividad & Correlación de Pearson & 1 & $.245 *$ \\
\hline & & Sig. (bilateral) & & .041 \\
\hline & & $\mathrm{N}$ & 70 & 70 \\
\hline & Bienestar psicológico & Correlación de Pearson & $.245 *$ & 1 \\
\hline & & Sig. (bilateral) & .041 & \\
\hline & & $\mathrm{N}$ & 70 & 75 \\
\hline
\end{tabular}

** La correlación es significativa al nivel 0,01 (bilateral).

* La correlación es significante al nivel 0,05 (bilateral).

De igual manera, usando el coeficiente de correlación de Gamma para variables ordinales, en todas las facultades, la relación es significativa entre las variables en estudio excepto en la Facultad de Ingeniería Industrial $(p>0.05)$.

Tabla N. ${ }^{0}$ 5. Correlación según facultades.

\begin{tabular}{lllcc}
\hline Facultad & & & Valor & $\begin{array}{c}\text { Sig. } \\
\text { aproximada }\end{array}$ \\
\hline Medicina Humana & Ordinal por ordinal & Gamma & .394 & .000 \\
& N de casos válidos & & 406 & \\
Derecho & Ordinal por ordinal & Gamma & .399 & .000 \\
& N de casos válidos & & 123 & \\
Educación & Ordinal por ordinal & Gamma & .514 & .000 \\
& N de casos válidos & & 99 & \\
Matematica & Ordinal por ordinal & Gamma & .370 & .000 \\
& N de casos válidos & & 228 & \\
Ingeniería Industrial & Ordinal por ordinal & Gamma & .201 & .060 \\
& N de casos válidos & & 120 & \\
Administración & Ordinal por ordinal & Gamma & .395 & .000 \\
& N de casos válidos & & 217 & \\
\hline
\end{tabular}


Por otro lado, también puede observarse que existe relación significativa entre el bienestar psicológico y el rendimiento académico, tanto en género como en las diferentes facultades, mientras que esta relación es significativa en los jóvenes y adultos, mas no en los adolescentes.

Tabla N. ${ }^{\circ}$ 6. Correlación entre Bienestar y rendimiento.

\begin{tabular}{lllcc}
\hline Sexo & & & Valor & $\begin{array}{c}\text { Sig. } \\
\text { aproximada }\end{array}$ \\
\hline Masculino & Ordinal por ordinal & Gamma & -.165 & .031 \\
& N. ${ }^{\circ}$ de casos válidos & & 358 & \\
Femenino & Ordinal por ordinal & Gamma & -.231 & .001 \\
& N. ${ }^{\circ}$ de casos válidos & & 474 & \\
\hline
\end{tabular}

Tabla N. ${ }^{0}$ 7. Correlación entre Bienestar y rendimiento según facultades.

\begin{tabular}{lllcc}
\hline Facultad & & & Valor & $\begin{array}{c}\text { Sig. } \\
\text { aproximada }\end{array}$ \\
\hline Medicina Humana & Ordinal por ordinal & Gamma & -.295 & .003 \\
& N de casos válidos & & 294 & \\
Derecho & Ordinal por ordinal & Gamma & -.269 & .034 \\
& N de casos válidos & & 107 & \\
Matematica & Ordinal por ordinal & Gamma & .099 & .387 \\
& N de casos válidos & & 217 & \\
Administración & Ordinal por ordinal & Gamma & -.246 & .013 \\
& N de casos válidos & & 214 & \\
\hline
\end{tabular}

Tabla N. ${ }^{\circ}$ 8. Correlación entre Bienestar y rendimiento según grupo etáreo.

\begin{tabular}{llcrc}
\hline Edad (Categorizada) & & Valor & $\begin{array}{c}\text { Sig. } \\
\text { aproximada }\end{array}$ \\
\hline Adolescente & Ordinal por ordinal & Gamma & -.187 & .281 \\
& N de casos válidos & & 94 & \\
Joven & Ordinal por ordinal & Gamma & -.201 & .001 \\
& N de casos válidos & & 684 & \\
Adulto & Ordinal por ordinal & Gamma & -.340 & .050 \\
& N de casos válidos & & 54 & \\
\hline
\end{tabular}

Cuando se observan conjuntamente la asertividad y el rendimiento académico, no hay asociación entre estas variables ni en el género ni en las diferentes facultades consideradas, ni en los diferentes grupos etáreos $(p>0.05)$. 
Tabla N. ${ }^{\circ}$ 9. Tabla cruzada entre asertividad y rendimiento según sexo.

Recuento

\begin{tabular}{|c|c|c|c|c|c|c|c|}
\hline \multirow[b]{2}{*}{ Sexo } & & & \multicolumn{5}{|c|}{ Asertividad (Categorizada) } \\
\hline & & & $<=-4$ & $-3-7$ & $8-19$ & $20+$ & Total \\
\hline \multirow[t]{4}{*}{ Masculino } & \multirow{3}{*}{$\begin{array}{l}\text { Rendimiento } \\
\text { académico }\end{array}$} & Tercio Superior & 15 & 23 & 26 & 18 & 82 \\
\hline & & Tercio Medio & 62 & 54 & 52 & 78 & 246 \\
\hline & & Tercio Inferior & 9 & 6 & 6 & 6 & 27 \\
\hline & Total & & 86 & 83 & 84 & 102 & 355 \\
\hline \multirow[t]{4}{*}{ Femenino } & \multirow{3}{*}{$\begin{array}{l}\text { Rendimiento } \\
\text { académico }\end{array}$} & Tercio Superior & 35 & 26 & 28 & 40 & 129 \\
\hline & & Tercio Medio & 84 & 71 & 82 & 75 & 312 \\
\hline & & Tercio Inferior & 6 & 3 & 4 & 6 & 19 \\
\hline & Total & & 125 & 100 & 114 & 121 & 460 \\
\hline
\end{tabular}

Tabla N. ${ }^{0}$ 10. Correlación entre asertividad y rendimiento según sexo.

\begin{tabular}{lllrc}
\hline Sexo & & & Valor & $\begin{array}{c}\text { Sig. } \\
\text { aproximada }\end{array}$ \\
\hline Masculino & Ordinal por ordinal & Gamma & -.036 & .633 \\
& $\mathrm{~N}^{\circ}$ de casos válidos & & 355 & \\
Femenino & Ordinal por ordinal & Gamma & -.048 & .520 \\
& N. ${ }^{\circ}$ de casos válidos & & 460 & \\
\hline
\end{tabular}

Tabla N. ${ }^{\circ}$ 11. Correlación entre asertividad y rendimiento según facultad.

\begin{tabular}{lllcc}
\hline Facultad & & & Valor & $\begin{array}{c}\text { Sig. } \\
\text { aproximada }\end{array}$ \\
\hline Medicina Humana & Ordinal por ordinal & Gamma & -.061 & .553 \\
& N de casos válidos & & 284 & \\
Derecho & Ordinal por ordinal & Gamma & -.105 & .482 \\
& N de casos válidos & & 103 & \\
Matematica & Ordinal por ordinal & Gamma & .070 & .487 \\
\multirow{2}{*}{ Administración } & N de casos válidos & & 216 & \\
& Ordinal por ordinal & Gamma & -.044 & .649 \\
& N de c asos válidos & & 212 & \\
\hline
\end{tabular}

Tabla N. ${ }^{0}$ 12. Correlación entre asertividad y rendimiento según grupo etáreo.

\begin{tabular}{llccc}
\hline Edad (Categorizada) & & & Valor & $\begin{array}{c}\text { Sig. } \\
\text { aproximada }\end{array}$ \\
\hline Adolescente & Ordinal por ordinal & Gamma & -.044 & .808 \\
& N de casos válidos & & 89 & \\
Joven & Ordinal por ordinal & Gamma & -.026 & .651 \\
& N de casos válidos & & 675 & \\
Adulto & Ordinal por ordinal & Gamma & -.182 & .311 \\
& N de casos válidos & & 51 & \\
\hline
\end{tabular}


No existe diferencia significativa en la asertividad, según sexo $(p=0.388)$, por lo que puede decirse que el sexo no está relacionado con la asertividad.

Tabla N. ${ }^{\circ}$ 13. Comparación de la asertividad según sexo de los sujetos.

\begin{tabular}{llrcc}
\hline & Sexo & N. $^{\text {o }}$ & $\begin{array}{c}\text { Rango } \\
\text { promedio }\end{array}$ & $\begin{array}{c}\text { Suma de } \\
\text { rangos }\end{array}$ \\
\hline Asertividad & Masculino & 542 & 592.44 & 321105.00 \\
& Femenino & 661 & 609.84 & 403101.00 \\
& Total & 1203 & & \\
\hline
\end{tabular}

Pero sí hay diferencia significativa en el bienestar psicológico según el sexo de los sujetos, $p<0.05$. Las mujeres tienen una puntuación mayor en promedio.

Tabla N. ${ }^{0}$ 14. Comparación del Bienestar psicológico según sexo de los sujetos.

\begin{tabular}{llccc}
\hline & Sexo & N. & $\begin{array}{c}\text { Rango } \\
\text { promedio }\end{array}$ & $\begin{array}{c}\text { Suma de } \\
\text { rangos }\end{array}$ \\
\hline Bienestar psicológico & Masculino & 547 & 563.59 & 308284.00 \\
& Femenino & 683 & 657.07 & 448781.00 \\
& Total & 1230 & & \\
\hline
\end{tabular}

Por otro lado, tampoco existe diferencia significativa en la asertividad y el bienestar psicológico según las facultades en estudio, mostrando una mayor asertividad los estudiantes de matemática.

Tabla N. ${ }^{\circ}$ 15. Comparación de la asertividad y bienestar psicológico según facultades de estudio.

\begin{tabular}{llcc}
\hline & Facultad & N. $^{\mathbf{o}^{*}}$ & $\begin{array}{c}\text { Rango } \\
\text { promedio }\end{array}$ \\
\hline Asertividad & Medicina Humana & 410 & 568.67 \\
& Derecho & 124 & 656.53 \\
& Educación & 104 & 600.22 \\
& Matematica & 228 & 669.57 \\
& Ingeniería Industrial & 120 & 517.95 \\
& Administración & 217 & 610.16 \\
& Total & 1203 & \\
Bienestar psicológico & Medicina Humana & 421 & 597.73 \\
& Derecho & 128 & 643.80 \\
& Educación & 113 & 680.74 \\
& Matematica & 229 & 586.57 \\
& Ingeniería Industrial & 120 & 559.58 \\
& Administración & 219 & 660.34 \\
& Total & 1230 & \\
\hline
\end{tabular}


Con respecto a los grupos etáreos, no se observa una diferencia significativa tanto en la asertividad como en el bienestar psicológico en función de la edad ( $p=0.155$ y 0.710 respectivamente), por lo que la edad no está relacionada con la asertividad ni con el bienestar psicológico), aunque los adultos muestran en promedio un mayor nivel de asertividad. Esta diferencia se debe al azar.

Tabla N. ${ }^{\circ}$ 16. Comparación de la asertividad y bienestar psicológico según el grupo etáreo.

\begin{tabular}{llrc}
\hline & Edad (Categorizada) & N. $^{\mathbf{0}}$ & $\begin{array}{c}\text { Rango } \\
\text { promedio }\end{array}$ \\
\hline Asertividad & Adolescente & 137 & 556.05 \\
& Joven & 996 & 605.05 \\
& Adulto & 70 & 648.55 \\
Bienestar psicológico & Total & 1203 & \\
& Adolescente & 142 & 638.68 \\
& Joven & 1013 & 612.39 \\
& Adulto & 75 & 613.61 \\
& Total & 1230 & \\
\hline
\end{tabular}

\section{CONCLUSIONES}

En general existe correlación significativa positiva entre el bienestar psicológico y la asertividad, siendo altamente positiva tanto en los varones como en las mujeres.

Respecto a la edad, esta relación también es positiva y altamente significativa en adolescentes y jóvenes, pero poco significativa en los adultos. Así, con el avance en la edad, la asociación entre el bienestar psicológico y la asertividad se va haciendo más débil. Esto es atribuible al hábito que aprende la persona experimentada en función a las consecuencias que obtiene comportándose asertivamente, siéndole indiferente si su entorno subjetivamente percibido es satisfactorio. También se verifica que en todas las facultades la relación es significativa entre las variables en estudio, excepto en la Facultad de Ingeniería Industrial.

El bienestar psicológico y el rendimiento académico se relacionan significativamente, tanto en género como en las diferentes facultades, en los jóvenes y adultos, pero no en los adolescentes. Esto llevaría a afirmar que las condiciones que producen la sensación subjetiva de bienestar proveen un contexto favorable para llevar a cabo tareas lectivas.

No se registra asociación entre la asertividad y el rendimiento académico, ni en el género ni en las diferentes facultades consideradas, ni en los distintos grupos etáreos, lo que se condice básicamente con el carácter de actividad solitaria que tiene el estudio, en la cual no se necesita ejercer habilidades de relación interpersonal.

No hay diferencia significativa en la asertividad, según sexo, por lo que puede decirse que el sexo no está relacionado con la asertividad. Pero sí hay diferencia significativa en el bienestar psicológico según el sexo de los sujetos. Las mujeres muestran una puntuación mayor en promedio, lo cual complementa los datos del estudio de Tarazona (2005) hecho 
con adolescentes escolares, donde los varones obtienen más índice de satisfacción vital que las mujeres (dejando en claro que en otros estudios [García y González, 2000], el bienestar y la satisfacción se correlacionan entre sí).

No se encuentra diferencia significativa en la asertividad y el bienestar psicológico según las facultades en estudio, aun cuando los estudiantes de matemática presentan un mayor cociente de asertividad.

Por último, referente a los grupos etáreos no se observa diferencias significativas ni en asertividad ni en bienestar psicológico en función de la edad, así que ésta no se halla fundamentalmente relacionada con la asertividad ni con el bienestar psicológico, pese a que los adultos muestran en promedio un mayor grado de asertividad.

\section{REFERENCIAS BICBLIOGRÁFICAS}

1. Alberti, R.E. y Emmons, M.L. (1974/2006). Con todo tu derecho: Asertividad e igualdad en su vida y en sus relaciones. Barcelona: Obelisco.

2. Aliaga, J. (2001). Variables psicológicas relacionadas con el rendimiento académico en matemática y estadística en alumnos del primer y segundo año de la Facultad de Psicología de la UNMSM. Revista de Investigación en Psicología, 4 (1). 35-52.

3. Atienza, F., Pons, D., Balaguer, I., y García-Merita, M. (2000). Propiedades psicométricas de la Escala de Satisfacción con la Vida en adolescentes. Psicothema, 12, 314-319.

4. Bequet B, Cortada de Kohan, N, Castro, A, y Renault, G. (2002). Factores que intervienen en el rendimiento academico de los estudiantes de psicología y psicopedagogía. USAL, 6, 18-36.

5. Díaz, J., y Sánchez, M. P. (2002). Relaciones entre estilos de personalidad y satisfacción autopercibida en diferentes áreas vitales. Psicothema, 14, 100-105.

6. Díaz, D., Rodríguez, R., Blanco, A., Moreno, B., Gallardo, I., Valle. C. y Van Dierendonck, D. (2006). Adaptación española de las escalas de bienestar psicológico de Ryff. Psicothema, 18(3), 572-577.

7. Diener, E., Suh, E. M., Lucas, R. E., y Smith, H. L. (1999). Subjective well-being: Three decades of progress. Psychological Bulletin, 125, 276-302.

8. Fensterheim, H. y Baer, J.L. (1975/2001). No diga sí cuando quiera decir no. México: Grijalbo.

9. Fierro, A. (2006). Bienestar personal, adaptación social y factores de personalidad: Estudios con las Escalas Eudemon. Clínica y Salud, 17(3), 297-318.

10. García, M. (1999). Estrategias de enseñanza de los valores y la autoestima. En Varios. La psicología educativa hacia el tercer milenio (pp.13-27). Lima: Universidad San Martín de Porres.

11. García, C. R. (2005). Habilidades sociales, clima social familiar y rendimiento académico en estudiantes universitarios. Liberabit, 11, 63-74.

12. García, V. y González, I. (2000). El bienestar psicológico. Su relación con otras categorías sociales. Revista Cubana de Medicina General Integral. 16(6): 586-592. 
13. Grande, I., Medina, P., Gomá, M. y Valero, S. (1998). Personalidad, diferencias individuales, y rendimiento académico en adolescentes. En M. Sánchez, M. Sánchez y M. Quiroga (Eds.). Perspectivas actuales en la investigación psicológica de las diferencias individuales (pp. 181-185). Madrid: Centro de Estudios Ramón Areces.

14. Inga, J. y Vara, (2006, 9 de ago.). Factores asociados a la satisfacción de vida de adultos mayores de 60 años en Lima. Ponencia presentada al V Encuentro Científico Internacional de invierno (08-11 de agosto). Lima-Perú.

15. Lange, A.J. (1980/1983). Entrenamiento cognitivo-conductual de la asertividad. En A. Ellis y R. Grieger (Dirs.). Manual de terapia racional emotiva (pp. 309-323). Bilbao: Descleé de Brouwer.

16. Medina, P., Grande, I., Gomá, M. y Valero, S. (1998). Personalidad, habilidades cognitivas e intereses profesionales en adolescentes. En M. Sánchez, M. Sánchez y M. Quiroga (Eds.). Perspectivas actuales en la investigación psicológica de las diferencias individuales (pp. 177-180). Madrid: Centro de Estudios Ramón Areces.

17. Montgomery, W. (1999). Asertividad, autoestima y solución de conflictos interpersonales. Lima: Círculo de Estudios Avanzada.

18. Novaez, M. (1986). Psicología de la actividad escolar. México: Iberoamericana.

19. Padrós, F. (2002). Disfrute y bienestar subjetivo. Un análisis psicométrico de la Gaudibilidad. Tesis para optar el grado de Doctor en Psicología. Universidad de Barcelona.

20. Pérez, M. P. (2000). Habilidades sociales: Educar para la autorregulación. Barcelona: Universidad de Barcelona.

21. Pizarro, R. (1985). Rasgos y actitudes del profesor efectivo. Tesis para optar el Grado de Magíster en Ciencias de la Educación. Pontificia Universidad Católica, Santiago de Chile.

22. Rathus, S. A. (1980). Un inventario de 30 ítems para evaluar el comportamiento asertivo. En R. Ardila (Ed.). Terapia del comportamiento (169-177). Bilbao: Descleé de Brouwer.

23. Reyes, Y. N. (2003). Relación entre el rendimiento académico, la ansiedad ante los exámenes, los rasgos de personalidad, el autoconcepto y la asertividad en estudiantes del primer año de psicología de la UNMSM. Tesis para optar el Grado de Psicólogo, UNMSM, Lima.

24. Salotti, P. (2006). Estudio sobre estrategias de afrontamiento y bienestar psicológico en una muestra de adolescentes de Buenos Aires. Tesis para optar el Grado de Lic. en Psicología, Universidad de Belgraño, Buenos Aires.

25. Tarazona, D. (2005). Autoestima, satisfacción con la vida y condiciones de habitabilidad en adolescentes estudiantes de quinto año de media. Un estudio factorial según pobreza y sexo. Revista del Instituto de Investigaciones Psicológicas UNMSM, 8(2), $57-65$.

26. Van-Der Hofstadt, C. J., Gómez, J. M., y Alarcón, M. J. (Eds.) (2006). Competencias y habilidades profesionales para universitarios. Madrid: Díaz Santos. 